Les sources de l'Histoire des Mines : Nouveaux outils, Nouvelles approches

\title{
La loi du 21 avril 1810 et le Conseil général des mines avant 1866 . Les procès-verbaux des séances
}

The law of 1810 and the General council of mines before 1866

\section{Lionel Latty}

\section{(2) OpenEdition}

\section{Journals}

Édition électronique

URL : http://journals.openedition.org/dht/803

DOI : $10.4000 /$ dht. 803

ISSN : 1775-4194

Éditeur :

Centre d'histoire des techniques et de l'environnement du Cnam (CDHTE-Cnam), Société des élèves du CDHTE-Cnam

Édition imprimée

Date de publication : 1 décembre 2008

Pagination : 17-29

ISBN : 978-2-95-30779-2-6

ISSN : 0417-8726

Référence électronique

Lionel Latty, « La loi du 21 avril 1810 et le Conseil général des mines avant 1866. Les procès-verbaux des séances », Documents pour l'histoire des techniques [En ligne], $16 \mid 2^{\mathrm{e}}$ semestre 2008, mis en ligne le 06 octobre 2010, consulté le 08 septembre 2020. URL : http://journals.openedition.org/dht/803 ; DOI : https://doi.org/10.4000/dht.803 


\title{
La loi du 21 avril 1810 et le Conseil général des mines avant 1866 Les procès-verbaux des séances
}

\author{
Lionel Latty \\ IDHE, Université Paris Ovest
}

\begin{abstract}
RésumÉ
La loi de 1810 fonde le droit minier en France. Elle accorde un véritable droit de propriété sur le tréfonds minéral au bénéfice du concessionnaire et organise la tutelle administrative de l'industrie minière et sidérurgique. Dans cet article, nous étudions l'origine de la loi et son application jusqu'à la déréglementation de l'industrie sidérurgique en 1866. L'accent est mis sur la comparaison avec l'œuvre législative de l'Ancien régime et de la Révolution et sur le rôle et l'action du Conseil général des mines, en particulier dans le domaine minier
\end{abstract}

Résumés et mots clés en anglais sont regroupés en fin de volume, accompagnés des mots clés français

a première moitié du XIXe siècle est caractérisée Lpar des mutations technologiques qui ont profondément modifié l'industrie et la société : besoins croissants en énergie avec un remplacement progressif du charbon de bois par la houille ; usage accentué des mécanismes hydrauliques, non seulement pour mettre en mouvement roues et turbines, mais aussi pour laver les minerais de fer, puis le charbon; développement de la machine à vapeur, d'abord pour épuiser les eaux souterraines des mines puis pour assurer la traction ferroviaire ; utilisation du coke en sidérurgie ; améliorations décisives des moyens de transports avec le plan canaux de Becquey à partir de 1823 et, peu après, avec le développement des chemins de fer ; recherches méthodiques du potentiel minier fondées sur de meilleurs connaissances géologiques ; exploitations plus rationnelles pour des travaux beaucoup plus profonds, exigeant l'extension des circuits d'aérage, de descente et remontée du personnel, de transport du minerai, et le stockage du stérile ; précautions à prendre pour lutter contre les explosions de grisou. Tous ces éléments introduisent des changements d'échelle, des concentrations vers les meilleurs gîtes houillers, des dangers nouveaux associés à l'indispensable amélioration de la productivité.

C'est dans ce contexte que les pouvoirs publics ont eu à exercer leur action. La loi du 21 avril 1810 confie en effet à l'administration des mines, à ses ingénieurs et son Conseil général de très larges pouvoirs de tutelle et de contrôle sur l'industrie minière. Nous examinerons ici successivement les origines de cette loi, ses principales caractéristiques et quelques aspects de son application au XIXe siècle, sous l'égide du Conseil général des mines '.

\footnotetext{
1 À l'origine de cette étude se trouve la réalisation d'un instrument de recherche conçu pour les Archives nationales [ensuite AN]: la table des matières enrichie des registres des procès verbaux du Conseil général des mines. Celle-ci concerne actuellement les 25 premiers registres manuscrits, de $F / 14 / 17920$ (registre tome $n^{\circ} 1$ ) à $F / 14 / 17944$ (registre tome $\left.n^{\circ} 25\right)$, correspondant à une période de 20 années, soit 907 séances, du 9 janvier 1811 au 6 septembre 1830 . Cet instrument de recherche est téléchargeable : $\underline{w w w}$. archivesnationales.culture.gouv. fr/chan/chan/pdf/sm/ PVCGMG\%E9n\%E9ral03.pdf. Une brochure téléchargeable est disponible pour l'utilisation de cet instrument de recher-
} 


\section{La loi du 21 avril 1810 et le Conseil général des mines}

\section{La législation d'Ancien Régime}

La loi sur les mines du 21 avril 1810 est à la fois un aboutissement et un renouvellement. Elle complète les dispositions prescrites par les quatre arrêts du Conseil du 9 août 1723 sur les fourneaux et les forges, du 15 janvier 1741 sur les mines métalliques, du 14 janvier 1744 et du 19 mars 1783 sur les mines de houille, dont elle améliore considérablement la mise en œuvre, et elle remplace la loi du $1791^{2}$.

L'arrêt de 1744, rendu sous l'influence de Trudaine, supprimait la liberté indéfinie laissée jusqu'alors aux propriétaires du sol de fouiller leurs fonds et d'y exploiter des mines de « charbon de terre » sans aucune autorisation préalable. II prescrivait aussi, pour la première fois, des mesures techniques importantes pour la sûreté, la solidité et la rationalité des exploitations souterraines. Les mines de houille étaient ainsi placées, comme toutes les autres mines métalliques du royaume, sous le régime des permissions ou concessions. C'était la fixation du principe même de la législation organisant les concessions, encore actuellement en vigueur avec des aménagements successifs mais qui n'en contredisent pas son fondement.

Ce principe directeur, que l'on retrouve dans la loi de 1810, est celui désigné sous le nom de droit régalien, l'un des trois principaux systèmes d'organisation de la propriété des mines, avec le droit d'accession et le droit domanial ${ }^{3}$. Héron de Villefosse et Migneron ont donné du droit régalien deux définitions opérationnelles complémentaires, le premier par son principe ${ }^{4}$, la primauté de l'État garant de l'intérêt général, le second par trois dispositions à respecter pour sa mise en œuvre ${ }^{5}$ : il s'agit de « régler la destination de la pro-

che : (www.archivesnationales.culture.gouv.fr/chan/chan/ pdf/sm/PVCGMntro.pdf).

La référence à cette source est indiquée dans cette étude par le tome, la page et la cote F/14 correspondante.

2 Lamé Fleury, De la législation minérale sous l'ancienne monarchie ou Recueil méthodique et chronologique des lettres patentes, édits, ordonnances, déclarations, arrêts du Conseil d'État du Roi, du Parlement, Paris, Auguste Durand, 1857.

3 Étienne Dupont, Cours de législation des mines, Paris, Dunod, 1881.

4 Héron de Villefosse, De la richesse minérale.... Introduction, p. 6 : le droit régalien en matière de mines est celui « que se réserve l'État entier, représenté par le souverain, de disposer de la propriété souterraine comme d'une propriété publique, indépendante de la propriété privée du terrain qui la recèle, et d'en disposer pour le plus grand avantage de la société 11 .

5 Migneron, « Droit des mines ॥, Annales des mines 1833 3s/t3 (p. 643) «Les formes de la législation ont souvent varié, mais on peut quant au fond la résumer dans la triple attribution priété souterraine » au bénéfice « des personnes qui peuvent le mieux la mettre en valeur », de « surveiller l'exploitation dans ses rapports avec l'ordre public, avec la conservation du sol, et avec la sûreté des ouvriers mineurs», de " percevoir un certain tribut sur les produits obtenus par l'exploitant $»$.

Les propriétaires du sol acceptent mal, principalement pour le charbon de terre, le principe de concessions attribuées à d'autres qu'à eux-mêmes, et qui leur font perdre la jouissance d'un tréfonds qu'ils ont commencé à exploiter comme une carrière, à partir d'affleurements, dans un terrain leur appartenant. Ils sont donc, en quelque sorte par principe et selon un réflexe d'appropriation de tout ce que contient «leur » terre, hostiles au droit régalien du tréfonds. Ces propriétaires du sol n'ont pas encore été confrontés à la nécessité de former des compagnies financières puissantes, indispensables pour passer du stade d'exploitations artisanales proches de la surface, à celles d'exploitations exigeant des travaux beaucoup plus profonds, attaqués par le bas et conduits méthodiquement vers les couches supérieures. L'incompréhension et l'opposition sont inévitables. II faudra l'inexorable constat de cette nécessité et non un principe juridique pour qu'ils évolvent.

Il faudra ainsi du temps et des compromis pour passer d'une industrie minière extractive embryonnaire, le plus souvent inexpérimentée dans un contexte de connaissances géologiques et scientifiques limitées, à une industrie changeant de taille et d'horizon, combinant de nouvelles techniques et d'amples moyens financiers, utilisant et profitant du développement de la machine à vapeur. Une opposition trop tranchée entre l'avant et l'après 1810 ne permet pas de comprendre le processus de changement d'une part, et la continuité des efforts d'autre part, pour achever ce qui a été commencé en 1744. La compréhension, les caractéristiques et les limites mêmes de la loi du 21 avril 1810 trouvent leurs sources dans cette période introductive. Dans le contexte politique des années 1790 et de la préparation de la loi de 1810, certaines grandes personnalités ont incontestablement minoré la portée des efforts de la réglementation de 1744 et de 1783.

Les exemples des bassins houillers du Forez et de la Loire présentent, de ce point de vue, des traits remarquables. L'opposition à la politique minière de l'État y est générale et de longue durée. Le droit d'accession s'y est imposé depuis longtemps indépendamment, puis contre le droit régalien. La disposition géologique

qu'elle conférait au prince ». 
y incite. Contrairement à l'Artois et la Flandre française, les couches de houille affleurent en bien des points, rendant extrêmement facile, tout au moins au début, d'en détacher le combustible ${ }^{6}$. Les propriétaires du Forez prennent ainsi l'habitude d'utiliser le charbon par des fouilles superficielles dans leurs terrains. II s'agit de leur charbon. Sur leur terre ils développent une pluriactivité artisanale, rurale et minière « d'extraction de carrières de charbon $\|^{7}$. L'exploitation stéphanoise, d'abord le fait d'une foule de petits propriétaires fonciers, se calque sur l'exploitation rurale ${ }^{8}$. La très forte résistance des propriétaires et extracteurs à l'arrêt de 1744 s'explique ainsi assez naturellement. Tout le Lyonnais est en proie à la fièvre judiciaire et les procès des propriétaires contre les concessionnaires se compliquent des contestations entre les propriétaires et leurs entrepreneurs extracteurs ${ }^{9}$. II n'est donc pas étonnant que les cahiers de doléances de ces provinces demandent l'abolition des concessions ${ }^{10}$. Ce que l'on appelle le privilège de Saint-Étienne favorise le repliement sur soi.

Avant 1789, arbitraire et favoritisme de Cour sont associés à l'idée de privilège. Les termes mêmes employés, de 1744 jusqu'en 1789, pour caractériser la nature des arrêts du Conseil d'État sur les mines peuvent donner prise à une telle représentation d'arbitraire. Un échantillon de 87 arrêts du Conseil du roi traitant des concessions de hoville montre ainsi que l'expression « droit d'exploiter » figure dans un seul cas situé dans le futur département de la Loire, à la date du 25 septembre 1779. Par contre celle de « privilège exclusif d'exploiter » est presque aussi fréquemment employée que celle de «permission d'ouvrir et d'exploiter exclusivement à tous autres». Les cahiers de doléances critiquent les «privilèges exclusifs » et demandent leur suppression. L'Essai sur les privilèges, publié en fin

6 Étienne Brossard, Études historiques sur la propriété, l'exploitation, et l'établissement des mines de houille dans le département de la Loire, Saint-Étienne, 1887, p.1.

7 Jean-Louis Alléon-Dulac, Mémoires pour servir à l'histoire naturelle du Forez, 2 vol., Lyon, 1765, tome 2, p. 2 : I'utilisation de ce mot "carrière " indique le type d'exploitation faite à partir de la surface du sol, et permet de comprendre l'origine de la représentation ancienne et constante que le Forez se fait des mines de charbon, inséparables du sol.

8 Louis Trénard, "Le charbon avant l'ère industrielle (17001800) "), dans id. éd., Charbon et sciences humaines, Paris-La Haye, EHESS/Mouton, 1966, p. 153-171.

9 Marcel Rouff, Les mines de charbon en France au XVIII siècle (1744-1791), Paris, Rieder, 1922, p. 128.

10 Louis de La Roque, Recueil des cahiers de 1789, Dauphiné, Forez, Lyonnais, Beaujolais, Paris, Dubuisson, 1867. d'année 1788 par l'abbé Sieyès, touche en son point nodal l'ancienne société et discrédite l'usage de l'expression « privilège ».

\section{La loi sur les mines de 1810}

La Révolution bouleverse les conditions du débat sur la propriété du sous-sol et sur la liberté d'en jouir. Rompant avec le système des privilèges, la loi du 28 juillet 1791 inspirée par Mirabeau, substitue à l'arbitraire du « privilège exclusif d'exploiter » le principe que «les mines et les minières sont à la disposition de la nation ». Leur exploitation exige désormais une permission préalable ou concession. Dans les faits, le droit d'exploitation est remis aux propriétaires de la surface, ce droit ne leur étant retiré que s'ils ne veulent ou ne peuvent exploiter. Plus que cette loi, les évènements affectent les conditions de l'exploitation. Dans le Nord, dès les premières années de la Révolution, et plus encore avec la guerre et l'invasion, commence pour les mines une longue période de crise ${ }^{11}$.

Au début, la situation est plutôt favorable à la Compagnie d'Anzin : la conjonction de la mise à la disposition de la nation des biens du clergé le 2 novembre 1789, et de la loi sur les mines du 28 juillet 1791 qui prolonge jusqu'à leur terme les concessions accordées par le Conseil du roi, lui permet d'acheter des biens nationaux enclavés dans ses possessions. Mais du fait de l'émigration de plusieurs actionnaires importants, les mines deviennent en majeure partie, propriétés de la République. Avec la guerre la situation s'aggrave. Beaucoup d'ouvriers quittent leur travail pour s'enrôler. La raréfaction de main d'œuvre minière provoque une diminution de l'extraction d'Anzin, la baisse des bénéfices de la Compagnie et un accroissement de son endettement. Enfin l'occupation par les troupes étrangères à mi juillet 1793 a deux lourdes conséquences : situation de disette de charbon « effrayante " ${ }^{12}$ |' hiver suivant et pillage de la région d'Anzin.

Dans le centre de la France, après le pillage de la concession de Roche-la-Molière, «les concessionnaires ne font plus exploiter à partir du 4 août 1789 ॥ ${ }^{13}$. Les registres de Rive-de-Gier sont brûlés sur l'ordre du représentant Javogues : « vous avez été longuement op-

11 Alexandre de Saint-Léger, Les mines d'Anzin et d'Aniche pendant la Révolution, Paris, E. Leroux, 1935, t. 1, 1e partie. 12 Arrêté du directoire du département du Nord le 2 août 1794 ( 5 thermidor an 2), A. de Saint-Léger, op. cit., pièce $n^{\circ} 33$ bis, $p$. 245: «considérant que la commune de Douai a éprouvé une disette effrayante de chauffage l'année dernière... ॥.

13 Séance CGM du 8 avril 1811 (†.1, p. 55, AN : F/14/17920). 


\section{La loi du 21 avril 1810 et le Conseil général des mines}

primés par une Compagnie de brigands, qui vous volaient impunément "), écrit-il à la municipalité le 22 septembre $1793^{14}$. Des décisions locales hostiles aux anciens concessionnaires et préjudiciables pour les exploitations sont prises par quelques administrations départementales.

À compter de l'été 1794, les mines et les forges, enjeu national pour soutenir l'effort de guerre, sont administrées par l'Agence des mines, devenu rapidement Conseil des mines. Le jeune corps des mines vient en appui technique de leurs dirigeants. Les missions dans les riches bassins miniers des territoires conquis amplifient l'expérience des ingénieurs français. De meilleures conditions sont réunies pour que puissent se dégager les principes directeurs et les modalités opérationnelles d'une politique minière et sidérurgique moderne ${ }^{15}$. Le Comité de salut public exprime dès la fin de l'année 1794 son souhait de voir « refondre, rectifier... les diverses lois rendues sur les mines et entre autres celle du 28 juillet 1791 1/ ${ }^{16}$. Le Conseil des mines formule des propositions législatives complémentaires en 1796. II souligne d'abord les conséquences dommageables de l'absence d'un principe constant concernant la propriété des mines ${ }^{17}$. II faut donc « une législation plus précise et d'après les principes constamment arrêtés sur la propriété des mines ». À cette date, le Conseil ne va pas plus loin sur ce sujet conflictuel. Mais il recommande « d'adopter le mode d'exploitation par des sociétés concessionnaires, dont les moyens puissent assurer des exploitations productives et durables, de réformer les abus attachés à la plupart des anciennes concessions de mines, qu'elles ne soient jamais un titre que pour exploiter avec activité une substance minérale quelconque, et dont l'étendue soit seulement et rigoureusement nécessaire à la prospérité de cet établissement ». Le mot « durable » tend à indiquer que la durée limitée à 50 ans par la

14 Camille Richard, Le Comité de salut public et les fabrications de guerre sous la terreur, Paris, Rieder, 1922, p.415.

15 Denis Woronoff, L'industrie sidérurgique en France pendant la Révolution et l'Empire, Paris, EHESS, 1984.

16 AN : F/14/1301/A Sous chemise marquée 43 au crayon "Loi sur les mines ", document marqué 41 au crayon.

1725 juillet 1796 (7 thermidor an 4), Compte rendu du Conseil des mines ; AN : F/14/1301/A : "L'intérêt particulier mal entendu, ou plutôt une ignorante cupidité et la charlatanerie se sont emparés des mines. Il en est résulté deux modes différents d'exploitation, l'un pour les concessionnaires obtenant du gouvernement le droit d'exploiter, l'autre par des propriétaires du sol, se prétendant propriétaires aussi des mines, et surtout sur les mines en couches sur lesquelles leurs vues se portent plus aisément». loi du 18 juillet 1791 ne convient pas. En reliant cette idée avec celle d'un principe constant concernant la propriété, la voie est ouverte à une réflexion sur la propriété du tréfonds accordée au concessionnaire. La synthèse et le compromis seront réalisés, sur ce point, par la loi de 1810.

L'arrêté du Directoire exécutif du 23 décembre 1797 (5 nivôse an 6) précise les conditions de cession des droits accordés par concessions et permissions d'exploiter les mines et les autorisations d'établir des usines. La loi du 2 février 1801 ( 13 pluviôse an 9) réduit de six mois (article 10 de la loi de 1791) à deux mois le délai accordé au propriétaire du sol pour manifester son intention de procéder ou non à l'exploitation des mines sous ses terrains aux mêmes conditions que celles imposées au concessionnaire non propriétaire. Cette disposition rend plus difficile pour le propriétaire l'élaboration et le financement d'un projet d'exploitation. L'instruction ministérielle du 7 juillet 1801 (18 messidor an 9) interprète la loi de 1791 au détriment des propriétaires du sol en fixant que ceux-ci ne peuvent plus disposer à leur gré - comme le prévoyait la fin de l'article 1 de la loi - des cent pieds sous la surface sans une permission du gouvernement, à demander et à obtenir suivant les formes prescrites par la loi pour l'octroi des concessions ${ }^{18}$. Cette instruction préfigure plusieurs dispositions de la loi de 1810 et de l'instruction ministérielle du 3 août relative à son exécution, en fixant les conditions préalables nécessaires pour qu'il y ait lieu d'accorder des concessions, telles que l'existence reconnue des minerais, la certitude de moyens offerts par les localités, l'étendue de la concession, la transmission à l'Administration des plans du gîte. Les rédactions sont même presque identiques.

La loi sur les mines du 21 avril 1810 remplace enfin la loi du 28 juillet 1791. La première version du projet de

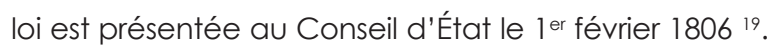
Quatre années ont été nécessaires pour parvenir au texte définitif. Les débats et controverses sont multiples au sein du Conseil d'État, particulièrement entre les partisans du droit régalien et ceux qui soutiennent le droit d'accession. Napoléon y prend une part importante. L'ouvrage de Locré en décrit les péripéties. Aguillon résume les moments forts des sept rédactions ${ }^{20}$. Cette élaboration longue et approfondie porte la marque des enseignements tirés depuis l'arrêt du 14 janvier

\footnotetext{
18 Louis Aguillon, Législation des mines..., op. cit., †. 1, p. 43 et Journal des mines $n^{\circ} 59$ : instruction 7 juillet 1801 .

19 lbid., p. 46.

20 lbid., p. 47 à 51.
} 
174421. II faut contourner les résistances de cette partie de la société civile rurale hostile au principe de droit régalien, comme le reste encore celle du Forez. II est essentiel que l'Administration puisse souverainement choisir le concessionnaire, sur des critères de compétence et de moyens financiers. II faut qu'elle soit elle-même agent de changement, par la diffusion des techniques appropriées et par la formation des élites techniques spécialisées. II faut rendre contradictoire l'instruction des demandes pour tempérer les critiques de choix arbitraires. II faut trouver un compromis sur la question de la propriété du tréfonds qui ne puisse pas entraver les objectifs précédents. Cet aboutissement et ce renouvellement mettent ainsi un terme, surtout pour les mines, à une longue période de règles contradictoires, révélatrices à la fois de l'évolution du rapport de force entre pouvoir gouvernemental et propriétaires terriens, d'une méconnaissance des caractéristiques géologiques des couches de minerai, allant jusqu'à l'hypothèse de «la reproduction ${ }^{22}$ "), et d'une hiérarchie entre les minerais nobles (or et argent), les minerais de fer pour la fonte au charbon de bois, et la houille (ou charbon de terre), reléguée longtemps à un rôle d'appoint local ${ }^{23}$.

Si la loi ne fait que réaffirmer le principe du droit régalien, elle diffère fondamentalement de celle de 1791 sur la question de l'exploitation. À partir de 1810, avec la préférence souveraine du gouvernement dans le choix du concessionnaire - cette disposition est essentielle - l'administration peut sélectionner parmi les différents demandeurs le groupe d'associés ou la personne qui lui semble le plus apte à réussir l'exploitation. La loi de 1810 attribue à tout concessionnaire, relativement au tréfonds, un titre de propriété incom-

21 Regnaud de Saint-Jean-d'Angély, Exposé des motifs du projet de la loi des mines, présenté au Corps Législatif le 13 avril 1810 .

22 E. Brossard, Études historiques, op. cit., cite (p. 128) un passage d'une lettre du 16 juillet 1709 de Charles Trudaine, intendant de la généralité de Lyon, à Desmarets, directeur des finances: " il y a une chose heureuse dans ces mines du Forez qui est la reproduction ; il y en a d'anciennement abandonnées pour avoir été épuisées, où l'on retrouve présentement du charbon qui s'est reformé depuis l'abandon $॥$. Brossard cite également une opinion identique de Gennete (ingénieur allemand) exprimée en 1774 dans Connaissance des veines de houille, et celle plus nuancée de Buffon, qui fixe certaines conditions.

23 Louis Trénard, "Le charbon avant l'ère industrielle ॥, op. cit. : " après 1744 on passe de la recherche dispersée à la constitution de sociétés; en 1810, le charbon entre peu à peu dans l'économie, mais scientifiquement et psychologiquement, il demeure un inconnu et un étranger $»$. mutable, que ne reconnaissait pas l'arrêt de 1744. En dépit de cette différence importante, la loi de 1810 est bien plus l'héritière des quatre arrêts de l'Ancien Régime cités que de celle du 28 juillet 1791, dès lors que le principe d'égalité des droits de la déclaration des droits de l'homme et l'instauration du nouveau Conseil d'État tendent à diminuer le risque d'arbitraire.

Il aura donc fallu vingt ans, concernant les mines, pour arriver au compromis de 1810. Celui-ci est favorable à la fois à la propriété et à l'État ${ }^{24}$. La propriété sort renforcée puisque par l'acte de concession une propriété perpétuelle nouvelle est créée. II est ainsi plus difficile, pour les adeptes du droit d'accession, de critiquer une loi qui permet d'ajouter un second potentiel de richesse et qui conforte les droits de tous les propriétaires, qu'ils le soient du fonds ou du tréfonds. En plus des droits que la loi reconnaît aux propriétaires de la surface, un sujet mal connu est celui de l'ampleur et des prix des acquisitions de terrains superficiels réalisés de gré à gré entre les deux parties (le concessionnaire et le propriétaire de la surface) permettant au premier de mettre fin aux indemnités à payer pour les dommages causés au sol et d'avoir les mains libres pour développer ses installations de surface comme il l'entend. De plus la liberté d'entreprendre est favorisée, et non le dirigisme. La pluralité des demandes crée les conditions de la concurrence. La préférence souveraine dans le choix du concessionnaire renforce l'État. La prise en compte de l'intérêt général est tempérée par les recours possibles devant les juridictions administratives, et par le rôle du Conseil d'État qui valide le plus souvent, tout au moins durant les premières décennies du siècle, les propositions de décrets ou ordonnances préparées par le Conseil général des mines.

Le vocabulaire de référence, en se stabilisant autour de l'expression "concession instituée ", concourt également à dissiper la critique d'arbitraire. Cette expression rappelle qu'une concession résulte d'une loi comportant des droits mais aussi des obligations pour ceux qui vont recevoir gratuitement la propriété d'une mine concédée. Mais le risque sousjacent de monopole - un autre type de privilège subsiste. Le Conseil des mines y est très attentif. Ce risque diminue avec la réduction ou le partage des périmètres. Mais il peut renaître par regroupement progressif de concessions, comme le montre dans les années 1840 la Compagnie des mines de la Loire. Et il en résultera le décret du 23 octobre 1852 interdisant la fusion de plusieurs concessions, sauf autorisation

24 R. de Saint-Jean-d'Angély, Exposé des motifs, op. cit. 


\section{La loi du 21 avril 1810 et le Conseil général des mines}

préalable obtenue dans les mêmes formes que pour l'institution des concessions, ce qui constitue une modification de l'article 31 de la loi de 1810.

En fin de compte, l'équilibre entre le droit de pleine propriété dont bénéficient actuellement les concessionnaires et leur devoir de respecter les mesures de police des mines a été rapidement rompu. Comme le révèlent les résistances rencontrées par l'administration des mines de la part des exploitants, et les décisions de justice favorables aux concessionnaires rendues dans les affaires d'accidents, la loi de 1810 a privatisé le tréfonds sans autre contrepartie que l'espoir d'une production permettant de répondre partiellement aux besoins de la consommation industrielle et privée.

Des oublis ont été identifiés dès l'origine : les concessions à l'abandon, les contraventions difficiles à sanctionner, le retard à donner une place importante dans les cahiers des charges à l'aérage, les mines de sel. Ils ne méritent pas, juge-t-on rapidement, que soit pris le risque de mettre à mal les compromis qui ont réussi à concilier l'intérêt de l'État, celui des concessionnaires, et celui des propriétaires du sol. Seules interviendront, pour les mines, quelques retouches législatives commandées par les circonstances, le 27 avril 1838 sur l'assèchement des mines, et le 27 juillet 1880 dans le contexte de la crise hovillère de 1872-1874.

Pour les usines à fer, la loi de 1810 ne comporte rien d'aussi essentiel et durable. « Les Forges, Fourneaux et Usines $"$ y figurent dans le titre VII, à une place modeste, connexe à celle des minières de fer. Huit articles traitent des permissions des usines, alors que la loi en comporte au total quatre-vingt seize. Dans la pratique, l'instruction des demandes relatives aux hauts fourneaux, affineries, lavoirs et patouillets dépassera celle des concessions pendant la première moitié du XIXe siècle. Ceci prendra beaucoup de temps à l'administration des mines, comme à l'administration des ponts et chaussées et au Conseil général des ponts et chaussées dès que l'usage des cours d'eau, navigables ou non, est en cause ${ }^{25}$. L'atomisation, puis la concentration, les nouvelles localisations, la grande variété des établissements sidérurgiques et leur évolution technique constituent par nature une situation très différente de celle des mines. La déréglementation, dans ce cas, sera inéluctable.

25 Le régime de l'eau en amont des prises est de la compétence des ponts et chaussées, les pollutions d'aval provoquées par les opérations de lavage des minerais de fer d'alluvions et leurs remèdes (bassins de décantation) sont de la compétence des mines.
Le Conseil général des mines : rôle et fonctionnement

En conséquence directe de la loi de 1810 sur les mines, le décret du 18 novembre 1810, qui organise le Corps des mines, institue le Conseil général des mines pour remplacer l'ancien Conseil des mines créé sous la Convention ${ }^{26}$. Le Conseil général des mines traite des mêmes sujets, mais son rôle n'a plus de portée exécutoire. Il est désormais seulement de conseil et d'influence. Le nouveau Conseil est animé par le même petit groupe d'hommes qui vient d'exercer des responsabilités hiérarchiques et opérationnelles considérables pendant les quinze années du Directoire, du Consulat et du début de l'Empire ${ }^{27}$. II est, avec le Directeur général des mines ${ }^{28}$ et son entourage, et avec les ingénieurs en service ordinaire dans les départements, un élément majeur de la mise en œuvre et de l'évolution de la politique minière de l'État pendant tout le siècle et de sa politique sidérurgique jusqu'à la déréglementation du 9 mai $1866^{29}$. Le contenu des procès-verbaux des séances du Conseil général des mines permet d'apprécier jusqu'à quel point et comment la loi de 1810 - avec ses principaux principes, ses déclinaisons réglementaires, son application qui n'est pas sans failles - a pu être un vecteur de développement économique et d'innovation technique, un instrument approprié pour ne pas entraver l'entreprise, pour faciliter les adaptations structurelles. Un autre objectif, celui de veiller à la sécurité du travail des mineurs, comportera en revanche des lacunes et des échecs.

Les séances du Conseil sont hebdomadaires et durent en moyenne trois heures. Le déroulement des séances est structuré en plusieurs parties : exposé général du sujet par le rapporteur ; résumé de tous les rapports émis par les administrations ayant réglementairement à donner un avis sur la question traitée (forêts, ponts-et-chaussées, préfets, ingénieurs en chef et ingénieurs ordinaires des mines) ; énoncé des de-

26 Étienne Dupont, Traité pratique de la jurisprudence des mines, Paris, Carillian, 1853. Ce décret du 18 novembre 1810 fait suite à la longue instruction ministérielle du 3 août 1810 relative à l'exécution de la loi du 21 avril 1810, reproduite dans Dupont, t. 2, p.79-102.

27 Les trois premiers inspecteurs généraux du Conseil général des mines sont Antoine Levebvre d'Hellencourt (1759-1813), Claude Lelièvre (1752-1835), François Gillet de Laumont (1747-1834).

28 Les trois personnalités marquantes sont Becquey, Legrand, Bigot de Boureuille.

29 Á partir de la loi du 9 mai 1866 une liberté réglementaire complète est rendue à l'industrie métallurgique qui rentre dans le droit commun de toutes les industries. 
mandes et des oppositions qu'elles suscitent avec une attention particulière portée au respect des procédures et des étapes de l'instruction, pour que celle-ci soit réellement contradictoire; proposition d'avis du rapporteur ; enfin avis du Conseil. Parfois le Conseil " est assemblé extraordinairement sur la convocation du directeur général, qui le mettra en comité lorsqu'il le jugera convenable ${ }^{30}$.

Durant ces vingt années, le Conseil général des mines se réunit ainsi 907 fois et examine 2348 dossiers. Le nombre de dossiers étudiés par séance varie de un à dix. Un quart des séances (231 séances) traite un seul dossier, signe d'un sujet considéré important ou difficile; un peu plus d'un quart (263 séances) traite deux dossiers ; un autre quart (227 séances) trois dossiers. Le département de la Loire suscite l'analyse de 131 dossiers. Cinq concessions houillères donnent lieu à plus de dix dossiers par site, les mines de fer de Rancié à vingt neuf. Enfin on peut estimer à environ 800 le nombre d'affaires distinctes examinées.

Les procès verbaux des séances n'existent qu'en un seul exemplaire original, alors que deux étaient prévus ${ }^{31}$. Pour marquer l'importance de leur contenu, ils " sont présentés au Directeur général, pour être par lui visés ${ }^{32}$. Leur texte intégral est confidentiel. Seules sont susceptibles de diffusion les conclusions en forme d'avis, adoptées en vue de l'établissement de règlements d'administration publique préparés par le Conseil d'État. Ces procès-verbaux ont donné lieu à quelques extraits imprimés, dispersés dans de nombreuses cotes de la série F/14 des Archives nationales ou reproduits dans les Annales des mines, mais avec parfois de malencontreuses coupures. Une estimation de ces extraits imprimés est de 15 à $20 \%$ du corpus source.

L'analyse des procès-verbaux permet de saisir la diversité et la complexité des sujets traités, de situer les acteurs, renouvelés ou non par la période révolutionnaire et impériale. Elle conduit à mesurer les étapes de transition entre l'économie préindustrielle de l'ancien régime et celle de l'industrialisation du

30 Article 47 du décret du 18 novembre 1810. Ces comités sont rares ; constitués à la discrétion du directeur général sur des sujets pouvant impliquer des enjeux politiques, ils visent à préparer la séance qui leur est consacrée ; le choix de ses membres révèle des tendances d'opinion qui se manifesteront lors des séances ordinaires, à l'issue desquelles, assez souvent, l'avis rendu n'est pas adopté à l'unanimité.

31 L'article 48 du décret du 18 novembre 1810 dispose : « Le secrétaire du Conseil général inscrira les délibérations sur deux registres ; l'un pour le Conseil, l'autre pour le comité». 32 Article 48 du décret du 18 novembre 1810.
XIX siècle. Elle facilite la compréhension du contexte spécifique des demandes et des oppositions qu'elles suscitent souvent. Elle révèle les motifs circonstanciés des décisions favorables et des rares rejets. Elle permet aussi d'affiner la compréhension de plusieurs sujets : évolution des esprits face à la nécessité de mettre en valeur et de moderniser le potentiel houiller, minier et métallurgique, représentation que ceux-ci se font de cette nécessité ${ }^{33}$; rôle des acteurs, déjà pionniers ${ }^{34}$ ou prenant avantage des opportunités de la période révolutionnaire et impériale, qu'ils soient apporteurs de capitaux, innovateurs en technologie, ou intervenants spécialisés; rythmes des étapes de transition entre l'économie préindustrielle de l'ancien régime et celle de l'industrialisation du XIXe siècle, en ce qui concerne les mines, principalement de houille, et la sidérurgie, principalement les hauts fourneaux et les affineries.

Durant les vingt premières années ${ }^{35}$, les avis les plus nombreux du Conseil général des mines (795) concernent d'abord les demandes en « permission pour l'établissement des Fourneaux, Forges et Usines ॥. Viennent ensuite les demandes en concession de mine (486). Ces avis sont rendus en forme de projets de décret ou d'ordonnance, et transmis au ministre de tutelle, pour être examinés, en tant que règlements d'administration publique, par le Conseil d'État. Les nombreux problèmes soulevés par le Titre $\vee$ «De l'exercice de la Surveillance sur les Mines par l'Administration " (art. 47, 48, 49, 50) provoquent 45 avis au titre de la "Surveillance " et 62 à celui des « Accidents". Les avis peuvent enfin traiter « tous les autres objets pour lesquels il sera jugé utile au service de connaître l'opinion du Conseil ${ }^{36}$. Ils concernent, selon les périodes, des questions de politique économique (droits de douane), de politique réglementaire (interprétations de jurisprudence, réformes de la loi sur les mines, loi sur le sel), des projets de statuts des sociétés anonymes dont l'objet social correspond aux exploitations visées par la loi d'avril 1810, des redevances, des rapports annuels d'activité du service ordinaire départemental.

33 L. Trénard, « Le charbon avant l'ère industrielle », op. cit., p. 81. 34 Desandrouin, de Croÿ et leurs associés (Taffin) (Anzin) ; Tuboeuf bassin d'Alais (Rouff, Marcel) ; Decazes bassin d'Aubin Aveyron ; Chagot Blanzy ; Fleur de Lix (Loire) ; Bert et Montcombroux (com. de Montcombroux-les-Mines) (bassin de Bert), Allier.

35 Durant la période 1811-1830, 795 avis « Usine ॥.

36 Avant dernier alinéa de l'article 46 du décret du 18 novembre 1810 . 


\section{La loi du 21 avril 1810 et le Conseil général des mines}

Cinq critères inspirent les avis du Conseil général des mines : $1^{\circ}$ promouvoir le progrès technologique ; $2^{\circ}$ discerner à quel moment et en quoi l'intérêt public doit l'emporter sur les intérêts particuliers; $3^{\circ}$ rendre compatibles et fructueux l'intervention de l'État, le protectionnisme et le libéralisme économique ; $4^{\circ}$ prendre en compte le capital humain, qu'il s'agisse de l'encadrement ou des conditions de travail des mineurs; $5^{\circ}$ éviter l'arbitraire par la confrontation contradictoire de multiples points de vue différents. Inspiration ne signifie pas efficacité, comme il est possible de l'expliquer lorsqu'il s'agit de la police des mines.

L'influence du Conseil s'explique par la conjonction de deux phénomènes. Ses avis concourent à apporter une réponse aux problèmes concrets de l'industrie houillère et de la sidérurgie, deux industries lourdes en gestation. Ils sont porteurs d'une expertise technique, motivée scientifiquement et exprimée à l'issue d'une instruction approfondie et assez nettement contradictoire. Cette influence comporte toutefois plusieurs risques : ingérence dans la gestion privée ; lenteur des décisions; confusion des rôles de conseil et de surveillance ; hésitations à sanctionner à temps les vices d'exploitation des concessionnaires, à partir des cahiers des charges ; interventions tardives rendues d'autant plus difficiles que les concessionnaires ont une puissance et un rôle croissant dans l'économie générale ; opposition du Conseil d'État; réflexe de défense de l'institution à laquelle on appartient. Ceci est perceptible quant il s'agit des améliorations à apporter à la législation minière ${ }^{37}$ ou quand il y aurait lieu d'aller plus au fond des choses pour déterminer les causes réelles de graves accidents miniers ${ }^{38}$. La Commission du grisou de 1876 illustre ce dernier sujet.

\section{Le problème de l'exploitation utile}

L'exploitation utile est un principe essentiel du droit minier français. L'instruction ministérielle du 3 août 1810 précise : « II y a lieu à demande de concession, lorsque le gisement des couches minérales est tellement reconnu qu'il y a certitude d'une exploitation utile». ॥ faut d'abord des recherches positives, ne se limitant pas aux affleurements. II faut également une connaissance assez précise de la géologie du gîte, de l'allure

37 Voir les nombreuses séances du CGM de 1847 à 1849 concernant les projets de réforme de 1847-1849

38 L'exemple de l'inondation de la mine de Lalle (Gard) du 11 octobre 1861 faisant 105 morts, séance CGM 20 juin 1862 (†.69 p.115, AN : F/14/17988) à comparer avec AN : $\mathrm{F} / 14 / 4200$. des couches et de leur constitution, de la qualité des minerais, et un plan général du site.

Preuve de la difficulté de sa mise en œuvre, il faut attendre la circulaire du 31 octobre 1837 pour une affirmation sans ambiguïté du principe :

" la première chose à faire pour solliciter une $<$ la $>$ concession d'un gîte minéral, et pour que l'administration puisse donner suite à la demande, c'est de justifier qu'il y a matière à concession ॥; ou encore : " ... que l'on ait d'abord constaté l'existence de la mine "), ou encore « que la première condition à remplir est de justifier qu'une mine existe ». Cette circulaire reconnaît qu'avant cette date il en a été souvent autrement : « ... des concessions sont demandées avant qu'on se soit assuré si des mines existent dans les terrains que l'on indique ; quelquefois ajoutant trop de créance à des découvertes annoncées prématurément, on a commencé l'instruction, fait des publications et affiches, et beaucoup d'inconvénients sont résultés de cette marche trop précipitée $\|{ }^{39}$.

L'enjeu est d'intérêt public. Des titulaires de concessions obtenues sans que l'existence du gîte soit constatée par des recherches suffisantes ${ }^{40}$ induisent des tiers en erreur en obtenant des capitaux considérables ${ }^{41}$ pour mettre en valeur des richesses dont l'existence n'est pas prouvée. Ce point est à l'évidence très important par ses conséquences. De telles concessions font également peser sur le sol une servitude sans objet, en principe contraire aux lois civiles ${ }^{42}$, mais cet aspect n'ayant pas la même portée est très peu évoqué. L'affaire du procès concernant Saint-Bérain ${ }^{43}$, une des quinze concessions de houille

39 Circulaire du 31 octobre 1837 sur les demandes en concession de mines, E. Dupont, Traité Pratique..., op. cit., t.2 Appendice p.253.

40 de Cheppe, "I Jurisprudence des mines ॥, Annales des mines, 1836, 3s/110-599.

41 La concession achetée $146.000 \mathrm{~F}$ en 1827 est apportée pour 3.500.000 F en 1837 à une société anonyme d'exploitation. Voir Lionel Latty, Henri Fournel 1799-1876, Ingénieur du corps des mines, saint-simonien, thèse de doctorat, Paris $\mathrm{X}$ Nanterre, 17 juin 2000.

$421^{\circ}$ Séance CGM 21 juin 1830 (t. 25 p. 253-261, AN : F/14/17944) concernant dossier demande en concession houille à Nans (Var) par Jourdan fils : malgré absence de tout renseignement sur la disposition du gîte, les avis du préfet et du rapporteur Beaunier sont favorables; I'avis du CGM est de surseoir à statuer ; $2^{\circ}$ Jurisprudence des mines, Annales des mines, 1834, 3s/ $\$ 5$, p. 679.

$431^{\circ}$ La Gazette des Tribunaux, dix articles entre le 21 juin et le 23 août 1838 ; $2^{\circ}$ Fournel, Henri, Rapport sur la houillè- 
du bassin du Creusot Blanzy, est une illustration frappante du premier point. Les accusés David-Samuel Blum et Cleemann sont condamnés pour escroquerie à trois ans de prison, 3.000 F d'amendes et réparation. Un deuxième exemple est celui de la concession des mines de cuivre de Mouzaïa, en Algérie, accordée les 22 septembre 1844 et 3 novembre 1846 à Elie de Montgolfier, Petit et Henry frères. C'est un désastre financier de quatre à cinq millions de francs. La vigilance du Conseil général des mines est, ici, réellement prise en défaut. L'administration des mines n'a pas su ou a omis d'exprimer un avis scientifique clair, dans un contexte où prévalait au sein du gouvernement une position politique volontairement optimiste à l'égard de l'Algérie ${ }^{44}$. Cinq séances du Conseil général des mines traitent spécifiquement du dossier Mouzaïa entre le 26 mai 1848 et le 16 mai 1856. C'est seulement lors de la séance du 10 juillet 1863 qu'il est officiellement reconnu que « les gîtes ne sont pas très riches ». L'inspecteur général Dussert conclura en 1932 à une erreur d'appréciation géologique ${ }^{45}$.

Les avis du Conseil général des mines sur les demandes de concessions illustrent cette difficile problématique de la «certitude d'une exploitation utile». Il en est ainsi pour plusieurs raisons. Le Conseil est partagé entre son exigence scientifique et l'enjeu économique et politique - du développement de la production de houille. II préfère souvent saisir l'occasion d'une mise en valeur rapide d'une exploitation plutôt que d'en retarder l'autorisation dans l'attente de recherches plus approfondies et d'une confirmation géologique des caractéristiques du gîte, ou enfin d'une chance renouvelée. Il est à la fois juge et partie. Ses membres, sont des scientifiques soucieux d'expérimentation critique. Mais, dans leur rôle d'administration, ils incitent à entreprendre. L'exercice est difficile pour parvenir à une synthèse objective. Le message retenu par les milieux d'affaires comporte moins de nuances. L'institution d'une concession est interprétée comme signifiant une future richesse et provoque une surenchère spéculative sur les cessions ou apports en sociétés qui s'ensuivent.

re de Saint-Bérain sur d'Heune, Paris, Everat, $1838 ; 3^{\circ}$ AN : F/14/7873, rapport du 6 août 1837 de l'ingénieur des mines Puvis au directeur général des ponts et chaussées et des mines Legrand.

44 Le dépouillement des Tableaux de la situation des établissements français en Algérie montre cet optimisme.

45 D. Dussertvet G. Bétier, Les mines et les carrières en Algérie, Paris, Genin, 1931, p. 298.
Les études préalables aux demandes en concession et aux transactions de cession ou d'apports impliquent souvent des ingénieurs du Corps. II peut s'agir d'expertises, expressément prévues par les articles 87 et 88 de la loi de 1810, d'arbitrages ou de conseils, fortement encouragés, car ils concourent à la diffusion des compétences de haut niveau. Ces ingénieurs sont en service ou en congé ${ }^{46}$. Leurs missions sont le plus souvent autorisées. Les incompatibilités sont limitées à la circonscription dont est chargée l'ingénieur, mais faut-il encore que ces missions soient toujours déclarées. II semble bien qu'elles sont plus nombreuses que ne le laissent croire les documents officiels ${ }^{47}$. Le risque d'une situation ambigüe existe. Celle-ci est la contrepartie négative de l'imbrication du monde industriel et des réseaux d'influence des ingénieurs des mines. Les missions d'ordre privé demandées à des Ingénieurs du Corps visent à inspirer confiance au public et leurs conclusions peuvent laisser prévoir ce que serait la position du Conseil général des mines. Il y a donc une certaine confusion entre l'état de membre du Corps et la fonction d'expertise recherchée. Michel Chevalier conseille David Blum pour les mines d'Epinac. Fournel exerce à titre privé, à la fin des années 1830, une fonction d'ingénieur conseil. II n'omet jamais à cette occasion de mettre en avant son état de haut fonctionnaire. En plusieurs circonstances ses prévisions optimistes, diffusées dans le public, sont contredites ultérieurement. Une autre situation peut se produire lorsqu'un ingénieur des mines exerce momentanément une fonction de direction pour une société et pèse de son autorité pour réfuter les conclusions d'un groupe d'experts. Tel est le cas de Charles Combes, directeur en 1825 de l'usine de Sainte-Marie-aux-Mines (Haut-Rhin). II réfute en février et octobre 1825 le rapport de quatre experts, dans le cadre d'une enquête de salubrité diligentée en novembre 1824 par le maire, qui conclut « à l'influence très nuisible, surtout pour les ouvriers, des vapeurs d'arsenic provoqués par le blanchissage de l'arsenic et le grillage du cuivre gris, même avec un procédé employant des fourneaux à condensateurs». Pour Combes «la santé des ouvriers ne devrait pas en souffrir $»$. Le Conseil général des mines autorise l'établissement sous condition ${ }^{48}$.

46 André Thépot, Les ingénieurs des mines du XIXe siècle. Histoire d'un corps technique d'État, tome l, 1910-1914, Éditions ESKA, Paris, 1998, p. 312.

47 Ibid., p. 347.

48 Séances CGM des 29 mai 1826 (t.19, p.554 à 568, F/14/17938) et 5 juin 1826 (†.20, p.1 à 3, F/14/17939). 


\section{La loi du 21 avril 1810 et le Conseil général des mines}

L'hypothèse de la continuité des couches, admise jusqu'en 1840, a provoqué de grandes erreurs d'appréciation du potentiel des gisements. Un rapport fait à l'Académie des Sciences en 1842 par Dufrénoy et Elie de Beaumont, tous deux membres du Conseil général des mines, soutient la première étude publiée sur ce sujet par Amédée Burat ${ }^{49}$ et souligne que :

"l'on suppose presque toujours une trop grande régularité aux terrains houillers... La hoville forme, dans certains cas, des couches continues et d'une épaisseur uniforme sur de grandes longueurs, comme cela a lieu dans le bassin de Newcastle, de Belgique et du Nord de la France, mais ce fait ne doit pas être généralisé. De grandes erreurs ont été commises sur le calcul de la richesse des terrains houillers, que l'on établit généralement en attribuant aux couches de houilles une puissance moyenne que l'on cube ensuite dans toute l'étendue du terrain $»$.

Jusqu'en 1840 des exemples montrent des calculs théoriques partant de cette hypothèse, appliquée à des affleurements reconnus à plusieurs kilomètres de distance.

Sur ce critère de « certitude d'exploitation utile » le Conseil général des mines tranche de façon variable. On se limitera ici à donner quelques exemples. Tantôt l'exigence conduit le Conseil à repousser la demande, à refuser le partage des concessions, à s'opposer à toutes les formules faisant disparaître l'unité dans la direction de l'exploitation. Tantôt une rigueur insuffisante se manifeste. II en est de même concernant le métal zinc, l'administration des mines autorisant l'ouverture de sites dont elle aurait dû conclure, par l'expertise, qu'ils étaient d'avenir incertain ${ }^{50}$.

Ainsi, durant la première moitié du XIXe siècle, malgré les dispositions de la loi de 1810 et de l'instruction ministérielle du 3 août 1810, les cas sont assez nombreux pour lesquels la certitude d'exploitation utile est présumée plus que démontrée.

\section{La question des concessions inexploitées}

L'inexploitation d'une concession est prévue par l'art. 49 de la loi de 1810, mais ne comporte pas de sanction, conformément au vœu de Napoléon. L'administration et le propriétaire du sol ont peu de

49 Amédée Burat, Mémoire sur le gisement de la houille dans le bassin de Saône-et-Loire, précédé du rapport fait à l'Académie des Sciences par MM. Brongniart, Elie de Beaumont et Dufrénoy, Paris, 1842, p. 6 et p. 7 ; Burat est professeur à l'École centrale.

50 Anne-Françoise Garçon, Mine et métal. Les non ferreux à l'épreuve de la modernité (1780-1880), Rennes, PUR, 1998, p. 133. moyens d'action devant l'inertie du concessionnaire. La section de l'Intérieur du Conseil d'État, consciente de cette difficulté, établit dès le 22 juin 1812 un projet de décret sanctionnant l'abandon des mines ou la cessation de travaux : "le concessionnaire d'une mine inexploitée depuis un an serait mis en demeure de reprendre les travaux dans le délai de six mois, et en cas d'inexécution, la mine serait vendue en justice $11^{51}$. Malheureusement ce texte, venant fort mal à propos dans le contexte de la campagne de Russie, n'a pas de suite. Vingt-cinq ans plus tard, la loi sur l'assèchement des mines du 27 avril 1838 organise en quelque sorte une expropriation pour cause d'utilité publique, fondée sur le refus de participer à des travaux de sécurité d'intérêt général. Mais les exploitations en chômage qui «n'inquiètent ni la sûreté publique ni les besoins des consommateurs» ne donnent lieu à aucune sanction et restent la propriété des concessionnaires ${ }^{52}$. II ne s'agit donc pas d'une déchéance, estimée en contradiction avec la pérennité de la propriété des mines voulue par l'article 7 de la loi de 1810.

La procédure de retrait de concession n'a été mise en œuvre que dans un nombre infime de cas, hors de proportion avec le nombre de concessions inexploitées. Étienne Dupont énumère en 1881 ces neuf cas, dont trois concernant l'Algérie ont été annulés par le Conseil d'État ${ }^{53}$. En 1883, 822 concessions sont inexploitées pour un total de 1328 soit $62 \%^{54}$. Ce nombre et ce pourcentage sont en augmentation constante ${ }^{55}$. La distinction entre mines épuisées après avoir été productives et mines jamais réellement productives n'est pas faite à ce stade de l'analyse. L'hypothèse est que cette lacune ne contredit pas l'observation suivante. Le nombre élevé de concessions inexploitées révèle a posteriori que beaucoup de concessions accordées n'ont pas été assez productives pour correspondre au principe de certitude d'exploitation utile. Se pose alors la question, mal résolve par la loi de 1810 : que faire à l'égard de ces concessions inexploitées devenant

51 E. Dupont, Traité pratique..., op. cit., t.1, p.68.

52 Circulaire du 29 décembre 1838 contenant des instructions pour la loi du 27 avril 1838, voir E. Dupont, Traité pratique..., op. cit., t.2, appendice, p. 246 à 253.

53 E. Dupont, Cours..., op. cit., p. 316.

54 Voir L. Aguillon, Législation..., op. cit., †. 2, nº 561 à n 567 , p. 33 (et de 29 à 35).

55 En 1855 : 358 mines inexploitées pour un total de 852 soit 42\% (Annales des mines, 1877, p. 244) ; en 1865 : 627 mines inexploitées pour un total de 1161 , soit $54 \%$ (E. Dupont, Cours..., op. cit., p. 317); en 1875 : 692 pour un total de 1235, soit 56\% (E. Dupont, Cours..., op. cit., p.317). 
sans objet, de leurs propriétaires et des propriétaires de la surface concernés?

La tentative du 10 février 1877 de procéder au retrait de toutes les mines inexploitées, dont les travaux ne seraient pas repris dans le court délai de deux mois, est immédiatement contredite par la circulaire du 15 juin $1877^{56}$. II faut attendre la circulaire du 24 avril $1891^{57}$ pour que l'administration « examine, une à une, chacune des concessions inexploitées, donne les motifs et donne son avis $॥$.

Cette circulaire dispose que, s'il n'y a pas de cause légitime à l'inexploitation, la déchéance est prononcée. Après quoi la mine est mise en adjudication. Si aucun acquéreur ne se manifeste, la concession est attribuée à l'Éłat. L'appréciation de la nature illégitime ou non de l'inexploitation est donc décisive. Trois exemples, extraits d'un échantillon de 50 dossiers examinés par le Conseil général des mines en novembre et décembre 1891, en application de la circulaire du 24 avril 189158, permettent de comprendre les fondements de cette appréciation ${ }^{59}$. Pour le premier, les mines de fer de Deveix, la cause ne pouvant être imputée au concessionnaire, il n'y a pas de déchéance, c'est donc reconnaître a posteriori une erreur du Conseil général des mines et du Conseil d'État. Dans le second exemple, la mine de hoville de Chadernac, il s'agit de l'épuisement du gîte, qui ne peut pas davantage être imputé au concessionnaire, il n'y a donc

56 Léon Michel, Recueil méthodique de légis/ation minérale, Saint-Étienne, 1896, pp.118-124.

57 Ibid., pp. 124-127.

58 Séances CGM (t. 121, AN : F/14/18040), dont la séance du 15 janvier 1892 (pp. 578-606) intéressante par le rappel des principes de la circulaire du 29 décembre 1838.

59 AN : F/14/18040 : 20 novembre 1891 (t. 121, p. 179,), mines de fer, Deveix à Sarroux, Corrèze, concession de 1840 au bénéfice de Pierre Mignot : deux filons a peu près verticaux de $70 \mathrm{~cm}$ de puissance, les travaux consistaient en une galerie de $45 \mathrm{~m}$ et deux petits puits abandonnés peu après l'ordonnance ; pour le rapporteur il s'agit d'un arrêt légitime des travaux, il n'y a donc pas de déchéance ; 27 novembre 1891 (t. 121, p. 222), houille, Chadernac, Haute-Loire, concession à Jourjon, Clair, Freydier (1849) : en chômage depuis 1855 , car la couche disparaît en profondeur, des travaux considérables sont effectués sans succès; pour le rapporteur, cette mine est inexploitée pour des causes légitimes, il n'y a donc pas de déchéance ; 27 novembre 1891 (†. 121, p. 228), mines de plomb et d'argent, Chazelles, Haute-Loire, concession à Mr Marie Brutus en 1848 : mine inexploitée depuis 1855 , les héritiers ayant refusé de payer la redevance sont déchus de leurs droits ; la mine est soumise à adjudication le 4 février 1875 ; aucun acquéreur ne se présente, la concession reste attribuée au domaine public. pas non plus de déchéance. Dans le troisième, c'est le défaut de paiement de la redevance fixe qui entraîne cette fois la déchéance. Ainsi, en fait, la déchéance n'est prononcée que si une concession inexploitée porte ou risque de porter préjudice à l'État ou à des tiers qui réclameraient.

Les cas de retrait définitif de concession sont extrêmement rares, six jusqu'en 1880, en excluant les trois arrêtés du ministre de la Guerre du 14 septembre 1849 concernant l'Algérie, annulés par arrêt du Conseil d'État du 24 juillet $1852^{60}$. Aucune déchéance n'a été prononcée par application directe de la loi de 1838. L'analyse détaillée des déchéances prononcées en conséquence de la circulaire du 24 avril 1891 sort du cadre de cette étude.

\section{Des réformes impossibles $d u \mathrm{XIX}^{\mathrm{e}}$ siècle, mais une déréglementation de la sidérurgie}

Le contraste est frappant entre les réformes de la sidérurgie et celles de l'industrie hovillère. Pour la première elle est totale, par déréglementation intervenant en une seule fois. Pour la seconde elle est impossible au XIXe siècle.

Pour la sidérurgie la réforme décisive est introduite par loi du 9 mai 1866 qui abroge les dispositions de la loi du 21 avril 1810, relative à l'établissement des forges, fourneaux et usines et aux droits établis à leur profit sur les minières du voisinage. Pour cette branche d'activité - qu'il s'agisse de la préparation des minerais (le lavage du minerai de fer d'alluvions posera bien des problèmes de pollution), de la production de la fonte, de l'affinage, de la transformation du fer et de la fabrication de l'acier - assez vite l'inutilité d'un dirigisme administratif se fait sentir. L'exposé des motifs de la loi du 9 mai 1866 montre le chemin parcouru depuis le début du siècle : "Le système de mise en tutelle de l'industrie métallurgique pouvait se comprendre au moment où cette industrie était naissante ..., où le gouvernement sentait le besoin de la développer à tout prix,... où les particuliers qui se livraient à cette industrie, peu éclairés, peu expérimentés peut-être, avaient besoin d'être protégés et guidés ${ }^{61}$. Désormais la sidérurgie s'inscrit dans la réglementation de droit commun des établissements insalubres et incommodes qui lui était déjà applicable.

60 Trois arrêtés du ministre de la Guerre (14 septembre 1849) visent, en Algérie, les concessions de mines de fer de Karésas, Bou-Hmara, Aïn-Morka. Le motif est l'inexploitation. Ces trois arrêtés sont annulés par le Conseil d'État le 24 juillet 1852.

61 Exposé des motifs de cette loi par le président de section du Conseil d'État Cornudet 


\section{La loi du 21 avril 1810 et le Conseil général des mines}

Par contre, il en est tout autrement pour l'industrie houillère. Au cœur de la problématique des réformes minières quatre questions sont essentielles : $1^{\circ}$ doit-on continuer à accorder les concessions à titre gratuit ou doit-on les vendre par adjudication au profit du Trésor public ? ; $2^{\circ}$ les cessions ou transferts de concessions doivent-elles être ou non autorisés, comme le prescrivait non pas la loi de 1791 mais l'arrêté du Directoire exécutif du 23 décembre 1797 (3 nivôse an 6) ; $3^{\circ}$ les réunions de concessions, susceptibles de constituer des monopoles doivent-elles être soumises à autorisation ? ; $4^{\circ}$ l'absence de toute exploitation ou le non respect du cahier des charges doit-il être réellement sanctionné par la déchéance d'une concession et son retrait ? Chacun de ces quatre sujets met en cause la propriété et introduit un changement dans le rôle de l'État à l'égard des entreprises hovillères. La loi de 1810 a été élaborée dans un contexte de compromis permettant d'écarter le principe d'accession favorisant les propriétaires du sol ; elle a créé pour le tréfonds une propriété perpétuelle, incommutable en faveur des concessionnaires, et a conféré à l'administration des mines un rôle voulu décisif dans le choix des concessionnaires et dans la surveillance des exploitations. II fut, plus tard, impossible de trouver un nouvel équilibre, une issue pour résoudre les problèmes rencontrés dans l'application de la loi de 1810. De surcroît, la période a présenté des incertitudes : I'une économique, dans les années 1840, avec la concentration en discussion des exploitations des mines de houille dans le Forez ; l'autre politique, en 1848, avec un changement de régime inachevé, marqué par une crise économique et des affrontements sociaux.

Six projets de réformes globales n'ont pas abouti au cours du XIXe siècle. Seul le projet ponctuel, mais important, de Vivien en 1848 sur les réunions de concessions, visant à faire obstacle au risque présumé de monopole de la Compagnie des mines de la Loire, prend corps quelques années plus tard avec le décret du 23 octobre 1852.

Sur ces six projets, les deux élaborés en 1847-1849 sont particulièrement intéressants. Jusqu'à cette date aucune modification importante n'avait été apportée ni même sérieusement proposée à la loi de 1810, sauf à certains égards la loi 27 avril 1838 sur l'assèchement des mines. Les causes de celle-ci, ses modalités d'exécution et ses conséquences expliquent d'ailleurs pour une part le mouvement de concentration des concessions du bassin houiller de Saint-Étienne.

Le premier projet préparé par les ordres du ministre des Travaux publics, Paul Jayr, en responsabilité du 9 mai 1847 au 18 septembre 1847, est vraisemblablement inspiré par son prédécesseur Dumon qui occupe durablement cette fonction (du 16 décembre 1843 au 9 mai 1847). Il est étudié par une Commission spéciale désignée le 29 novembre 1847, dont son président est Cordier. Celui-ci est aussi le vice-président du Conseil général des mines. Cordier critique le projet et propose un contre-projet qui écarte toutes les mesures novatrices. II entraîne le Conseil général des mines dans le même sens, au cours des quatre séances tenues durant la première quinzaine de janvier $1848{ }^{62}$. Le second projet est celui de la Commission d'exploitation des mines désignée par le ministre des Travaux publics Marie le 3 mai 1848, dont le président est l'ingénieur des mines saint-simonien Jean Reynaud ardent propagandiste de la « Doctrine » à Lyon en mai 1831 avec Pierre Leroux ${ }^{63}$. Ce projet largement débattu, faisant une synthèse estimée acceptable par le gouvernement, est adopté par le Conseil après corrections au cours de onze séances tenues entre janvier et juin $1849{ }^{64}$. Avec le projet Jayr ou celui de Jean Reynaud, il s'agit d'une refonte entière de la législation de 1810. Les modifications assez profondes sont ressenties comme trop radicalement contraires aux principes admis en 1810. Ces deux tentatives sont réunies dans la version corrigée par le Conseil général des mines d'un nouveau projet. Cette version ultime est adoptée en partie par la section de législation, mais elle échove définitivement en 1849 devant l'assemblée générale du Conseil d'État ${ }^{65}$.

Vingt-cinq ans plus tard, un nouveau projet (une refonte complète) est élaboré par Lamé Fleury dans le contexte de la crise houillère de 1872-1875. Ce projet donne lieu à un avis très réservé du Conseil d'État. II se traduit finalement, a minima, par la loi du 27 juillet 1880 portant seulement sur des points mineurs : réduction de la servitude de protection accordée aux maisons d'habitation, diminution de la durée des enquêtes lors de l'instruction des demandes de concessions, régularisation de la procédure d'occupation des terrains à l'intérieur du périmètre, faculté d'ouvrir des travaux de secours ou des voies de communication en dehors

62 Séances CGM des 7, 12, 14 et 15 janvier 1848 (†.45, p. 506 à 531, AN : F/14/17964), totalisant une vingtaine d'heures de délibération.

63 Lettre de Reynaud à Charton du 12 mai 1831, BA FE 7645/141.

64 Séances CGM des 19 et 25 janvier, 9 février, 2 mars, 16 mars, 3 avril, 21 mai, 25 mai, 1, 4 et 15 juin 1849 (t. 47, une trentaine de pages entre p. 144 et p. 340, AN : F/14/17866). 65 L. Aguillon, Législation..., op. cit., t. 1, p.59. 
de ce périmètre, extension et définition plus explicite de la surveillance administrative des mines. Enfin, trois autres projets restent sans suite législative : celui du 25 mai 1886, du 5 mai 1894, et enfin du 23 mars 1900 présenté par le ministre des Travaux publics, Pierre Baudin.

Avec le temps, de nombreuses petites concessions continuent de s'épuiser, le contexte social et politique évolve, de nouveaux enjeux miniers apparaissent. Ce qui avait été considéré comme impossible dans le cadre de la loi de 1810 durant tout le XIXe siècle devient réalité. Ainsi dans les années 1910 deux dispositions législatives reviennent à supprimer l'appropriation privée de la mine et à consacrer l'appropriation par l'État. La première, le 13 juillet 1911, fixe que «les mutations de propriété, sous quelque forme et à quelque titre que ce soit, et les amodiations de concessions minières ne peuvent être effectuées que si elles ont été autorisées par un décret rendu sur avis conforme du Conseil d'État ॥. La seconde prescrit le 9 septembre 1919 pour toutes les nouvelles concessions (les concessions antérieures conservant le régime ancien) par l'article 1 qu'« il ne sera plus accordé de concessions de mine que pour une durée limitée et avec participation aux bénéfices, de l'État et du personnel», et par l'article 2 que « la durée de la concession, sera fixée par le cahier des charges invariablement à 99 ans pour les gisements de houille ou lignite, et à cinquante ans au minimum et à 99 ans au maximum pour les autres gisements $॥$.

En conclusion de cette étude, qui a mis l'accent sur les mines sans pour autant en traiter tous les aspects, quatre points se dégagent.

Une poignée de jeunes ingénieurs, confrontés aux difficultés non résolves de la plupart des exploitations hovillères à la fin de l'Ancien Régime, accrues par la Révolution et les premières années de guerre, soutenus par le pouvoir politique et portés par l'exemple des réussites minières étrangères, ont voulu et su trouver le plus souvent, avec le Conseil d'État, les voies et moyens de dégager un ferme compromis quant aux principes et modalités devant régir la mise en valeur du potentiel minier national, moins naturellement favorable que celui de l'Angleterre, de la Belgique et de la Prusse.

La loi de 1810 s'explique et trouve ses racines dans la situation de retard des exploitations houillères de la France du XVIIII siècle, exception faite de la très grande réussite de la Compagnie d'Anzin, et du redressement amorcé par l'arrêt du Conseil de janvier 1744. II s'est agi de trouver une réponse au très grave problème énergétique résultant de la rareté croissante du bois et de son renchérissement, à l'aube de la période active d'industrialisation lourde. Le désenclavement des marchés locaux, le développement de l'usage des machines à vapeur, les transports ferroviaires ont été des facteurs généraux de productivité. Toutefois, les tentatives pour réformer la législation n'ont pas réussi à prendre en compte substantiellement la transformation des exploitations minières, les conditions de travail des mineurs, l'influence dominante des principales sociétés houillères, les évolutions sociales de la société.

En troisième lieu, dans les trente dernières années du siècle, les nouvelles techniques d'abattage, l'extension et la profondeur croissante des champs d'exploitation, l'augmentation de la productivité - tous éléments par ailleurs indispensables pour faire face à la pression concurrentielle et prendre en compte la structure géologique peu favorable de nombreux gîtes houillers à l'exception du Nord et du Pas-de-Calais - ont créé une situation mal appréciée et mal résolve, dans laquelle la dangerosité des multiples nouveaux facteurs de la production s'est accrue et dans laquelle les conditions de salubrité de travail des mineurs de fond ne pouvaient plus être ignorées. Se pose ainsi la question de la maîtrise du progrès technique, particulièrement avec la catastrophe de Courrières et ses 1100 morts, le 10 mars 1906.

Enfin, le Conseil des mines, puis le Conseil général des mines, peuvent apparaître, le plus souvent, comme l'expression d'une magistrature scientifique et technique. Mais celle-ci n'est pas à l'abri de critiques. 